\title{
Quantification of resilience to water scarcity, a dynamic measure in time and space
}

\author{
S. P. Simonovic and R. Arunkumar \\ Department of Civil and Environmental Engineering, \\ University of Western Ontario, London - N6A 5B9, Canada \\ Correspondence to: S. P. Simonovic (simonovic@uwo.ca) \\ Published: 12 May 2016
}

\begin{abstract}
There are practical links between water resources management, climate change adaptation and sustainable development leading to reduction of water scarcity risk and re-enforcing resilience as a new development paradigm. Water scarcity, due to the global change (population growth, land use change and climate change), is of serious concern since it can cause loss of human lives and serious damage to the economy of a region. Unfortunately, in many regions of the world, water scarcity is, and will be unavoidable in the near future. As the scarcity is increasing, at the same time it erodes resilience, therefore global change has a magnifying effect on water scarcity risk. In the past, standard water resources management planning considered arrangements for prevention, mitigation, preparedness and recovery, as well as response. However, over the last ten years substantial progress has been made in establishing the role of resilience in sustainable development. Dynamic resilience is considered as a novel measure that provides for better understanding of temporal and spatial dynamics of water scarcity. In this context, a water scarcity is seen as a disturbance in a complex physical-socio-economic system. Resilience is commonly used as a measure to assess the ability of a system to respond and recover from a failure. However, the time independent static resilience without consideration of variability in space does not provide sufficient insight into system's ability to respond and recover from the failure state and was mostly used as a damage avoidance measure. This paper provides an original systems framework for quantification of resilience. The framework is based on the definition of resilience as the ability of physical and socio-economic systems to absorb disturbance while still being able to continue functioning. The disturbance depends on spatial and temporal perspectives and direct interaction between impacts of disturbance (social, health, economic, and other) and adaptive capacity of the system to absorb disturbance. Utility of the dynamic resilience is demonstrated through a single-purpose reservoir operation subject to different failure (water scarcity) scenarios. The reservoir operation is simulated using the system dynamics (SD) feedback-based object-oriented simulation approach.
\end{abstract}

\section{Introduction}

Risk, resilience, and vulnerability are the fundamental characteristics that defines the state of a system and are widely used to assess the performance of the system. Among these indices, resilience refers to the systems capability to recover from a failure. The concept of resilience was first introduced by Holling (1973) in ecological systems and defined as the measure of ability of the system to absorb changes and still persist with same basic structure when subjected to stress. Further, Hashimoto et al. (1982) extended the application of resilience to water resources system. They defined resilience as the measure that describes how quickly a system will likely to recover or bounce back from failure once failure has occurred. Over the period of time, the concept of resilience has been implemented in various domains. Thus, there are various definitions of resilience in different fields. Bruneau et al. (2003) reported in the context of earthquake engineering that the measure of system resilience should show the reduced failure probability, reduced consequences and reduced time to recovery. Haimes (2009) defined resilience as the ability of the system to withstand a major disruption within acceptable degradation parameters and to recover within an 
acceptable time and composite costs and risks. Vugrin et al. (2010) defined resilience as the ability of a system to efficiently reduce both the magnitude and duration of the deviation of system performance from the targeted system performance levels. Ayyub (2014) mentioned resilience as the ability of the system to return to a stable state after a perturbation. Based on the concepts of Bruneau et al. (2003), various dynamic measures were reported for assessing the system's ability to bounce back from a failure state, as seen above.

Though, various approaches were developed for estimating the resilience of the system based on the concepts of Bruneau et al. (2003), most of the approaches are estimating the resilience as a time independent measure that do not provide much insight about the recovery capability of the system over time. Also, most of these measures are domain specific and a number of issues must be taken into consideration while applying in other domains. The time independent static resilience is merely an abstract attribute of the system and does not completely describe the state of the system composed of vectors specific to any sub-state. Thus, the time independent static resilience measures are practically ineffective for efficient planning and developing appropriate system recovery strategies from a failure. Simonovic and Peck (2013) first developed a framework to quantify the resilience as a dynamic measure through system dynamics (SD) simulation approach and demonstrated the concept for the coastal urban flooding caused due to climate change. The developed framework considers the economic, social, organizational, health and physical impacts of climate change.

The main objective of this study is implementation of the framework developed by Simonovic and Peck (2013) for quantifying the resilience to a water scarcity (deficit) scenario with consideration of variability in time and space. The implementation is illustrated using simulation of reservoir operation for irrigation. The rest of the paper is organized in the following manner. The quantitative space-time dynamic resilience measure (STDRM) is presented in the next section. The presentation of the SD simulation of STDRM for a single-purpose reservoir operation is then presented. Results of the reservoir simulation and its space time dynamic resilience are then discussed.

\section{Space-time dynamic resilience}

The quantitative resilience measure, first introduced by Simonovic and Peck (2013) following Cutter et al. (2008), has two qualities: inherent (functions well during nondisturbance periods); and adaptive (flexibility in response during disturbance events) and can be applied to physical environment (built and natural), social systems, governance network (institutions and organizations), and economic systems (metabolic flows). An original space-time dynamic resilience measure (STDRM) of Simonovic and Peck (2013) is

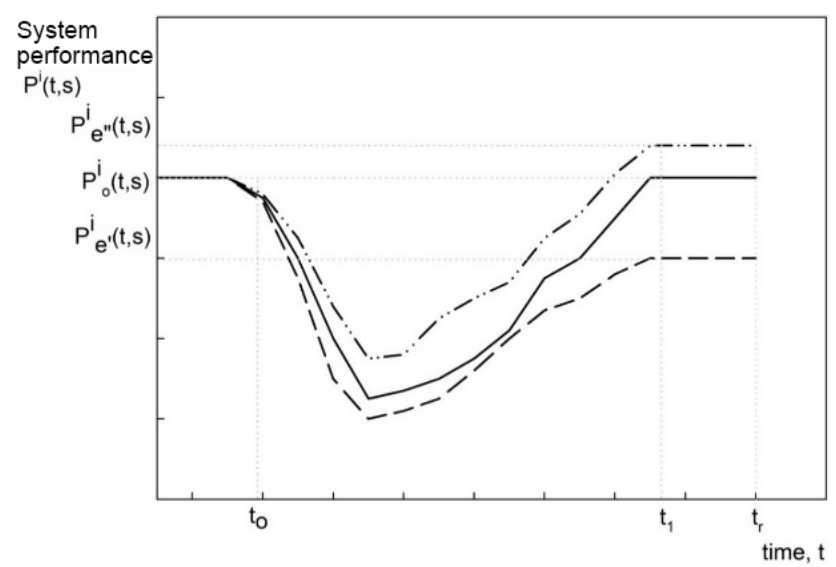

Figure 1. System performance.

designed to capture the relationships between the main components of resilience; one that is theoretically grounded in systems approach, open to empirical testing, and one that can be applied to address real-world problems in various domains.

STDRM is based on two basic concepts: level of system performance and adaptive capacity. They together define resilience. The level of system performance integrates various impacts $(i)$ of system disturbance. The following impacts (units of resilience $\left(\rho^{i}\right)$ ) can be considered: physical, health, economic, social and organizational, but the general measure is not limited to them. Measure of system performance $P^{i}(t, s)$ for each impact $(i)$ is expressed in the impact units (for example physical impact may be length [km] of road being inundated; and so on). This approach is based on the notion that an impact, $P^{i}(t, s)$ which varies with time and location in space, defines a particular resilience component of a system under consideration, see Fig. 1 adapted from Simonovic and Peck (2013). The area between the initial performance line $P_{0}^{i}(t, s)$, and performance line $P^{i}(t, s)$ represents the loss of system performance, and the area under the performance line $P^{i}(t, s)$ represent the system resilience $\left(\rho^{i}(t, s)\right)$. In Fig. $1, t_{0}$ denotes the beginning of the disturbance, $t_{1}$ the end of disturbance and $t_{\mathrm{r}}$ the end of the recovery period.

In mathematical form, the loss of resilience for impacts $(i)$ represents the area under the performance graph between the beginning of the system disruption event at time $\left(t_{0}\right)$ and the end of the disruption recovery process at time $\left(t_{\mathrm{r}}\right)$. Changes in system performance can be represented mathematically as:

$\rho^{i}(t, s)=\int_{t_{0}}^{t}\left[P_{0}^{i}-P^{i}(\tau, s)\right] \mathrm{d} \tau$ where $t \in\left[t_{0}, t_{\mathrm{r}}\right]$

When performance of the system does not deteriorate due to disruption, $P_{o}^{i}(t, s)=P^{i}(t, s)$ for an impact $(i)$, the loss of resilience is 0 (i.e. the system is in the same state as at the 


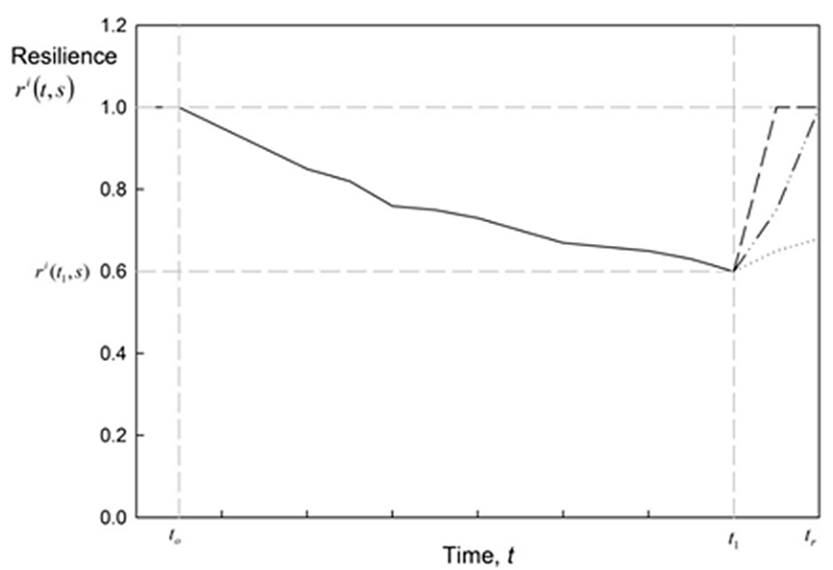

Figure 2. System resilience.

beginning of disruption). When all of system performance is lost, $P^{i}(t, s)=0$, the loss of resilience is at the maximum value. The system resilience, $r^{i}(t, s)$ is calculated as follows:

$r^{i}(t, s)=1-\left(\frac{\rho^{i}(t, s)}{P_{o}^{i} \times\left(t-t_{o}\right)}\right)$

As illustrated in Fig. 1, performance of a system which is subject to a disaster event drops below the initial value and time is required to recover the loss of system performance. Disturbance to a system causes a drop in system resilience from value of 1 at $t_{o}$ to some value $r^{i}\left(t_{1}, s\right)$ at time $t_{1}$, see Fig. 2. Recovery usually requires longer time than the duration of disturbance. Ideally, resilience value should return to a value of 1 at the end of the recovery period, $t_{\mathrm{r}}$ (dashed line in Fig. 2); and the faster the recovery, the better. The system resilience (over all impacts $(i)$ ) is calculated using:

$R(t, s)=\left\{\prod_{i=1}^{M} r^{i}(t, s)\right\}^{\frac{1}{M}}$

where $M$ is total number of impacts.

The calculation of STDRM for each impact $(i)$ is done at each location $(s)$ by solving the following differential equation:

$\frac{\partial \rho^{i}(t)}{\partial t}=\mathrm{AC}^{i}(t)-P^{i}(t)$

where $\mathrm{AC}^{i}$ represents adaptive capacity with respect to impact $i$.

The STDRM integrates resilience types, dimensions and properties by solving for each point in space $(s)$ :

$\frac{\partial R(t)}{\partial t}=\mathrm{AC}(t)-\prod_{i} P^{i}(t)$

The implementation of the presented framework is proceeding by using system dynamics simulation approach together with spatial analysis software.

\section{Space-time dynamic resilience to water scarcity}

In the present study, the space-time dynamic resilience of a simple single-purpose reservoir has been quantified using SD simulation approach. To achieve this, the operations of the single-purpose reservoir and its dynamic resilience is modelled using Vensim package - a SD simulation software (Ventana Systems, 2005).

\section{Reservoir simulation model}

The simple single-purpose reservoir simulation model consists of the continuity equation and a set of operational constraints. The continuity equation is expressed as:

$S_{t}=S_{t-1}+I_{t}-\mathrm{IR}_{t}-O_{t}-\mathrm{SP}_{t}$

where $S_{t}$ is the final storage at the time period $t ; S_{t-1}$ is the initial storage in the reservoir; $I_{t}$ is the inflow during the time period $t ; \mathrm{IR}_{t}$ is the total irrigation release from the reservoir during the time period $t ; O_{t}$ are the losses from the reservoir (evaporation and other leakage losses); and $\mathrm{SP}_{t}$ is the spill from the reservoir during the time period $t$. The system constraints, reservoir operating rules and the release decisions are captured using IF-THEN-ELSE statements in the simulation model. If the available storage is greater than the irrigation demand, then the actual demand is released; else the available storage is released. Then, the releases to the individual fields are made sequentially based on their demand.

The system performance for the reservoir irrigation $\left(P_{t, s}\right)$ for each individual field $s$ at time $t$ is expressed as the ratio of actual release made for irrigation and the demand during the time $t$ :

$P_{t, s}=\frac{\mathrm{IR}_{t, s}}{\mathrm{IR}_{t, s}^{\text {demand }}}$

where $\operatorname{IR}_{t, s}^{\text {demand }}$ is the irrigation demand during the time period $t$ for the field $s$. This performance measure is used for quantifying the space-time dynamic resilience.

\section{An illustrative case study}

Data required for the simulation are taken from Arunkumar and Jothiprakash (2012). Ten years of historical daily inflow observed at the dam site was collected and used for simulation. Analysis of inflow data shows that the reservoir is highly intermittent in nature and receives inflow only during the monsoon season. Inflow during the non-monsoon season is highly negligible. The reservoir supplies irrigation water to the command area at the downstream through the lift irrigation scheme. More details about the reservoir system can be found in Arunkumar and Jothiprakash (2012).

The illustrative model is set to include 100 individual fields to be irrigated by the reservoir. All individual fields are assumed to have equal area and same crop, therefore the 


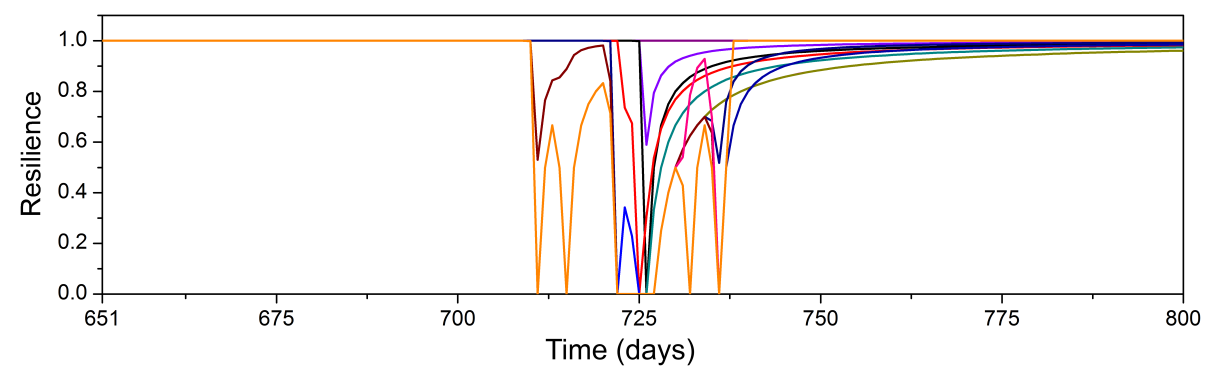

Figure 3. Temporal variation of resilience to irrigation water scarcity.
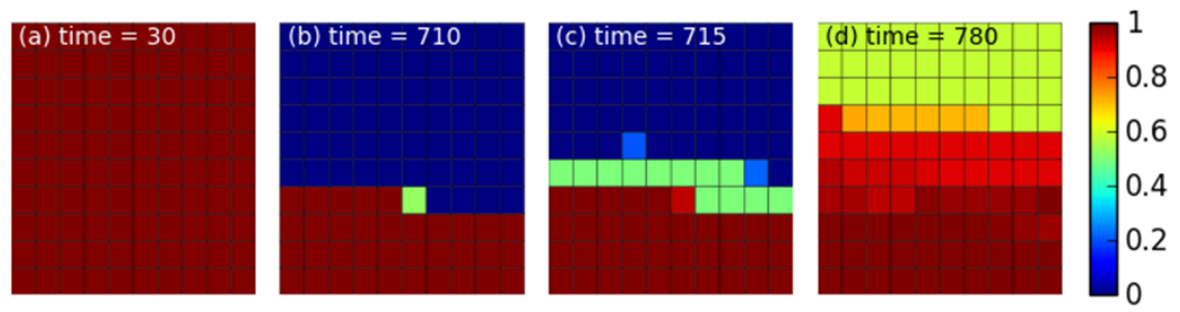

Figure 4. Spatial variation of resilience to irrigation water scarcity.

all fields have same irrigation demand. Water from the reservoir is delivered according to the distance from the reservoir, closer fields get the water first. However, it is be noted that the demand of individual fields may vary depending on crop type, plot size, etc. and release schedule can be modified to accommodate any other order of priority. In the illustrative case, the releases are made sequentially starting from field 1 , which is closest to the reservoir finishing with filed 100 being furthest away from the reservoir. Thus fields which are closer to reservoir get irrigated frequently and with increase in distance from the reservoir, irrigation frequency decreases. In event of inadequate water availability, all the fields may not be irrigated to their full demand. In such case, the available water is released to the fields closer to the reservoir. The system performance is estimated individually for each field (using Eq. 7).

The computational procedure integrates (a) reservoir operation and space time dynamic resilience models simulation using system dynamics approach in Vensim and (b) spatial irrigation release distribution model in Python. Integrated system provides for space-time dynamic resilience calculation of single-purpose reservoir operations.

\section{Simulation experiment}

The reservoir operation is simulated for 10 years using observed inflow and specified daily irrigation demand. Both, the inflow and the irrigation demand vary significantly on daily time scale. The reservoir receives predominant inflow during the monsoon season. However, the irrigation demand during monsoon season is insignificant due to the availability of rainfall. The irrigation demand is very high during non- monsoon seasons, but water availability is low. Thus, the system is prone to failure during non-monsoon season. Hence, the capability to recover from failure due to water scarcity is studied using SD approach.

\section{Simulation results}

The dynamic resilience of all 100 fields for the time period 651 to 800 is shown in Fig. 3. It is observed that initially all fields receive sufficient amount of water resulting in high resilience for most of the time. When the water availability is not sufficient to meet the demands, failure occurs. Then, the resilience of individual fields vary significantly and drops due to either partial satisfaction of the demand or no satisfaction. During the failure periods, due to lower amount of water being available, not all the fields receive the full demand. Only the fields near the reservoir receive the full demand, one field receives partial demand and most of the fields do not receive any water. Hence, there is variation in performance value and resilience with time and in space. It is observed from the Fig. 3 that the rate of recovery of induvial fields also vary. Some fields have higher rate of recovery and hence their resilience value suddenly increases from zero. These fields are closer to the reservoir, which are more resilient when failure happens. Fields far away from the reservoir exhibit much slower recovery and are less resilient.

The spatial dynamic resilience of all 100 individual fields is shown in Fig. 4 for four selected time periods. Figure 4a shows resilience of all fields at early part of the simulation period, day 30. During the initial periods, irrigation demand of all individual fields is fully met and therefore they have high resilience value. However, during non-monsoon season, 
due to less inflow in to the reservoir, irrigation demand are satisfied for only few fields. Due to the simple assumptions that the water from the reservoir is delivered in accordance to the distance (first the closets and so on) some fields closer to the reservoir get their demand fully satisfied whereas fields further away do not. Figure $4 \mathrm{~b}$ shows the spatial distribution of resilience at day 710. The fields closer to the reservoir always receive sufficient amount of water and therefore show high resilience. Most of the fields further away fail to receive the irrigation releases and hence their resilience drops to zero. Figure $4 \mathrm{c}$ shows day 715 where some of the fields already recovered from failure and their resilience increased. Figure $4 \mathrm{~d}$ shows day 780 when almost all fields are receiving the water for irrigation and their resilience is on the rise. As it can be seen from Fig. 4, the resilience of individual fields varies significantly. This is due to difference in the performance value and failure time of each field. Thus, their rate of recovery also varies. Due to our assumption, the fields closer to the reservoir have always higher resilience then fields further away.

\section{Discussion}

In this study, the concept of space-time dynamic resilience is applied to reservoir operation for the water scarcity scenario. The developed framework of the dynamic resilience is based on the original work of Simonovic and Peck (2013). Irrigation is the reservoir purpose used to measure the performance of the reservoir operation. Less reservoir inflow and insufficient storage resulted in changes in system performance and dynamic resilience. Temporal and spatial presentation of resilience can be used as the mechanism to investigate various adaptation options by repeating the simulations for various scenarios. In this way space-time resilience can provide for more efficient and better informed decision making process.

\section{References}

Arunkumar, R. and Jothiprakash, V.: Optimal Reservoir Operation for Hydropower Generation using Non-linear Programming Model, J. Inst. Eng. (India) Ser. A, 93, 111-120, doi:10.1007/s40030-012-0013-8, 2012.

Ayyub, B. M.: Systems Resilience for Multihazard Environments: Definition, Metrics and Valuation for Decision Making, Risk Anal., 34, 340-355, doi:10.1111/risa.12093, 2014.

Bruneau, M., Chang, S. E., Eguchi, R. T., Lee, G. C., O’Rourke, T. D., Reinhorn, A. M., Shinozuka, M., Tierney, K., Wallace, W. A., and von Winterfeldt, D.: A Framework to Quantitatively Assess and Enhance the Seismic Resilience of Communities, Earthq. Spectra, 19, 733-752, doi:10.1193/1.1623497, 2003.

Cutter, S. L., Barnes, I., Berry, M., Burton, C., Evans, E., and Tate, E.: A place-based model for understanding community resilience to natural disasters, Global Environ. Chang., 18, 598606, doi:10.1016/j.gloenvcha.2008.07.013, 2008.

Haimes, Y. Y.: On the Definition of Resilience in Systems, Risk Anal., 29, 498-501, doi:10.1111/j.1539-6924.2009.01216.x, 2009.

Hashimoto, T., Stedinger, J. R., and Loucks, D.: Reliability, Resiliency, and Vulnerability Criteria for Water Resource System Performance Evaluation, Water Resour. Res., 18, 14, doi:10.1029/WR018i001p00014, 1982.

Holling, C. S.: Resilience and Stability of Ecological Systems, Annu. Rev. Ecol. Syst., 4, 1-23, doi:10.1146/annurev.es.04.110173.000245, 1973.

Simonovic, S. P. and Peck, A.: Dynamic Resilience to Climate Change Caused Natural Disasters in Coastal Megacities Quantification Framework, Br. J. Environ. Clim. Chang., 3, 378-401, doi:10.9734/BJECC/2013/2504, 2013.

Ventana Systems: Vensim User's Guide. Ventana Systems, Inc., Belmont, MA, USA, 2005.

Vugrin, E. D., Warren, D. E., Ehlen, M. A., and Camphouse, R. C.: A Framework for Assessing the Resilience of Infrastructure and Economic Systems, in: Sustainable and Resilient Critical Infrastructure Systems, edited by: Gopalakrishnan, K. and Peeta, S., Springer Berlin Heidelberg, Berlin, Germany, 77-116, doi:10.1007/978-3-642-11405-2_3, 2010. 